\title{
Open thoracoabdominal aortic repair for chronic type B dissection
}

\author{
Nicholas T. Kouchoukos, MD, ${ }^{\mathrm{a}}$ Alexander Kulik, MD, MPH, ${ }^{\mathrm{b}}$ and Catherine F. Castner, RN, BSN ${ }^{\mathrm{a}}$
}

Objectives: Advances in endovascular surgery have brought into question the role of open operative treatment of chronic thoracoabdominal aortic dissection. In this context, we evaluated our experience with open repair of this condition using a single operative technique.

\begin{abstract}
Methods: From January 1986 to January 2014, 69 patients with chronic thoracoabdominal aortic dissection underwent open repair using total cardiopulmonary bypass (CPB) and hypothermic circulatory arrest (HCA). The degree of repair was as follows: Crawford extent I, 13 patients (19\%), Crawford extent II, 41 patients (59\%), and Crawford extent III, 15 patients (22\%). Thirty patients (43\%) had Marfan or Loeys-Dietz syndrome. Fifty-three patients $(77 \%)$ had previous operations on the thoracic or abdominal aorta.
\end{abstract}

Results: The 30-day mortality rate was 5.8\% (4 patients). Stroke occurred in $2(3 \%)$ of 66 operative survivors, and spinal cord ischemic injury in $4(6 \%)$. Temporary dialysis for new-onset renal failure was required in $4.5 \%$ of hospital survivors and tracheostomy in $10.6 \%$. Survival after 1,5 , and 10 years was $87 \%, 65 \%$, and $40 \%$, respectively. Eighteen patients $(26 \%)$ required a total of 20 subsequent operations on the thoracic or abdominal aorta of whom 15 had Marfan or Loeys-Dietz syndrome. Three of these procedures were for contiguous distal aortic disease and 10 were for patch aneurysms of the intercostal or visceral/renal arteries.

Conclusions: Open thoracoabdominal aortic repair for chronic dissection using CPB and HCA can be accomplished with mortality and morbidity rates that are comparable with those reported for endovascular or hybrid techniques. Open repair should remain a viable and primary option for the management of this condition until the long-term effectiveness of alternative methods of treatment is clearly established. (J Thorac Cardiovasc Surg 2015;149:S125-9)

As a result of improved management of acute aortic dissection with open or endovascular repair and medical treatment, the number of patients presenting with chronic dissection of the descending thoracic and thoracoabdominal aorta is increasing. The optimal management strategy for patients with chronic dissection of the thoracoabdominal aorta who require intervention is not clearly established. Until recently, open repair represented the only surgical option. However, endovascular and hybrid repair of chronic thoracoabdominal aortic dissection are being used with increasing frequency, bringing into question the role of open surgery as the method of choice. In this context, we reviewed our experience with open repair of chronic thoracoabdominal aortic dissection using total cardiopulmonary bypass $(\mathrm{CPB})$ and hypothermic circulatory arrest (HCA).

From the Division of Cardiovascular and Thoracic Surgery, ${ }^{a}$ Missouri Baptist Medical Center, St Louis, Mo; and the Division of Cardiothoracic Surgery, ${ }^{b}$ Lynn Heart Institute, Boca Raton Regional Hospital, Boca Raton, Fla.

Disclosures: Nicholas T. Kouchoukos reports consulting fees from Kips Bay Medical. All other authors have nothing to disclose with regard to commercial support.

Read at The American Association for Thoracic Surgery Aortic Symposium,

New York, New York, April 24-25, 2014.

Received for publication April 24, 2014; revisions received June 23, 2014; accepted for publication July 16, 2014; available ahead of print Sept 8, 2014.

Address for reprints: Nicholas T. Kouchoukos, MD, Cardiac, Thoracic, and Vascular

Surgery, 3009 N. Ballas Rd, Suite 360C, St Louis, MO 63131 (E-mail: ntkouch@ aol.com).

$0022-5223 / \$ 36.00$

Copyright (c) 2015 by The American Association for Thoracic Surgery

http://dx.doi.org/10.1016/j.jtcvs.2014.07.064

\section{MATERIALS AND METHODS \\ Patient Characteristics}

Between January 1986 and January 2014, 274 patients underwent open surgical repair of the descending thoracic or thoracoabdominal aorta repair using total CPB and HCA. No other operative technique was used during this interval. Sixty-nine of these patients $(15 \%)$ had chronic thoracoabdominal aortic dissection and constitute the study population. The clinical characteristics of the 69 patients are shown in Table 1 . The mean age at the time of operation was $54 \pm 16$ years (range, 22-77 years), and 53 $(77 \%)$ patients were male. Thirty patients $(43 \%)$ had clinical manifestations of Marfan or Loeys-Dietz, syndrome. Thirty-three patients (48\%) had symptoms associated with their aortic disease. The remaining patients had aneurysms greater than $5.5 \mathrm{~cm}$ in maximum diameter or rates of growth of the dissected aorta greater than 4 to $5 \mathrm{~mm}$ per year. Fifty-four patients $(78 \%)$ had undergone previous operations on the thoracic or abdominal aorta (Table 1). Seventeen patients had undergone 2 or more procedures.

The extent of aortic disease requiring replacement is shown in Table 1. Patients with Crawford extent IV disease, where the aneurysmal segment was confined to the abdominal aorta, were excluded from the analysis. The study was reviewed by the Institutional Review Board of the Missouri Baptist Medical Center and was exempted from the need for board approval.

\section{Operative Technique}

Our general technique using total CPB with an interval of HCA has been described previously. ${ }^{1,2}$ With increasing experience, several modifications have been introduced. Since September 2004, cerebral spinal fluid drainage (CSF) has been used routinely, if technically feasible. Because of concern for embolization of thrombus or atheroma into the cerebral circulation with the use of femoral artery perfusion during CPB, in 1997, we began inserting a dispersion arterial cannula (Edwards Lifesciences Inc, Midvale, Utah) into the descending thoracic aorta at a site free of thrombus or atheroma as determined by epiaortic ultrasonography. CPB is established with this 


\section{Abbreviations and Acronyms \\ $\mathrm{CPB}=$ cardiopulmonary bypass \\ $\mathrm{CSF}=$ cerebral spinal fluid \\ $\mathrm{HCA}=$ hypothermic circulatory arrest \\ $\mathrm{SCII}=$ spinal cord ischemic injury}

arterial cannula and with femoral venous cannulation. Femoral artery perfusion is initiated only after the aorta distal to the segment to be replaced has been occluded with a clamp. Alternatively, an 8-mm collagen impregnated polyester graft is anastomosed to the left axillary artery through a small incision in the axilla, and CPB is initiated through this artery.

After adequate cooling is achieved, the proximal anastomosis of the aortic graft to the descending thoracic aorta is completed during an interval of HCA. Flow is then reestablished to the upper body. Patent intercostal and lumbar arteries below the sixth or seventh intercostal space are preserved, when technically feasible. A full-thickness cuff of aortic tissue containing the intercostal arteries is sutured to an opening in the aortic graft. The clamp present on the aortic graft is repositioned below the implanted intercostal/lumbar arteries and perfusion of these arteries is established.

Before 2003, the visceral and renal arteries were attached to the aortic graft as a Carrel patch by using a full-thickness cuff of aortic tissue surrounding these arteries. Often, the left renal artery was attached separately to the aortic graft using a 6-mm or 8-mm interposition graft. Because of the development of patch aneurysms, particularly in patients with Marfan or other connective tissue syndromes, we modified our technique. In the subsequent 29 patients, the celiac, superior mesenteric, and renal arteries were detached from the aorta and attached separately to the branches of a woven polyester branched aortic graft (Hemashield Platinum TAAA graft, MAQUET Cardiovascular LLC, Wayne, NJ). ${ }^{3}$ Anastomosis of the body of the branched graft to the aorta distal to the renal arteries was performed before attaching the renal and visceral arteries to the aortic graft, to permit perfusion of the lower body through the second arterial line in the femoral artery. This was done to minimize the duration of spinal cord ischemia. The cardiopulmonary perfusion data are shown in Table 2.

\section{Follow-up}

After hospital discharge, patients were evaluated at 1 and 6 months postoperatively and subsequently at 12 -month intervals. Computed tomography or magnetic resonance imaging studies were obtained before discharge, at 6 months postoperatively, and yearly thereafter. Follow-up was $100 \%$ complete. Mean duration of follow-up was 5.4 years (range, 1 month to 19.4 years). Twenty-nine patients were followed for more than 5 years.

\section{Statistical Analysis}

Data were analyzed using Intercooled Stata 9.2 software (StataCorp, College Station, Tex). Standard descriptive statistical analyses were used. Continuous data are presented as a mean \pm standard deviation. Categorical data are presented as proportions and were compared between subgroups by using the Fisher exact test. Nonparametric estimates of freedom from all-cause death and reoperation were determined using the Kaplan-Meier method and are reported as mean \pm standard errors.

\section{RESULTS}

\section{Early Mortality}

The 30-day mortality rate was $5.8 \%$ (4 patients). Three patients died in the immediate postoperative period: 2 patients died intraoperatively of myocardial failure, and the third died early after arrival in the intensive care unit of tension pneumothorax resulting from perforation of the trachea. A fourth patient died of multiple organ system failure on the 18th postoperative day. Two additional patients died in the hospital of multiple organ system failure 83 days and 108 days postoperatively. The 1-year actuarial mortality rate was $13.4 \%$. Mortality according to the extent of aorta replaced is summarized in Table 3.

\section{Neurologic Complications}

Spinal cord ischemic injury (SCII) occurred in $4(6.1 \%)$ of 66 operative survivors. All developed paraplegia SCII according to the extent of aorta replaced is shown in Table 3. SCII occurred in $1(2.4 \%)$ of 42 patients who had implantation of intercostal or lumbar arteries, and in $3(12.5 \%)$ of 24 patients in whom no intercostal or lumbar arteries were implanted. The difference in the incidence of paraplegia was not statistically significant $(P=.13)$. Two of 22 patients $(9 \%)$ who underwent CSF drainage developed paraplegia and are alive 4.5 and 6 years later. Two of 44 patients $(4.5 \%)$ who did not undergo CSF drainage developed paraplegia. They survived for 18 days and 4.5 years. This difference was not statistically significant $(P=.6)$. Stroke occurred in $2(3 \%)$ of the operative survivors. The incidence of stroke according to the extent of aorta replaced is summarized in Table 3.

\section{Other Morbidity}

New-onset renal failure requiring dialysis developed in 3 $(4.5 \%)$ patients. Ventilatory support was required for a mean of $6.6 \pm 16.2$ days (median, 1.5 days), and 15 patients $(23 \%)$ required ventilatory support for longer than 72 hours. Tracheostomy was required in $7(10.6 \%)$ patients. The mean duration of stay in the intensive care unit was $10.2 \pm 19.6$ days (median, 4 days). The average intraoperative transfusion requirements were 8.3 units of packed red cells, 6.7 units of fresh frozen plasma, 3.8 units of platelets, and 10.2 units of cryoprecipitate. Two patients $(2.8 \%)$ required early reoperation for bleeding.

\section{Long-Term Follow-up}

During the follow-up period, which extended to 18 years, there were 31 late deaths. Two of the 21 patients whose cause of death was known, died of rupture. Five- and 10year actuarial survival was $65 \%$ and $40 \%$, respectively (Figure 1). Five- and 10-year actuarial rates of freedom from reoperation on the contiguous aorta were $97 \%$ and $83 \%$, respectively (Figure 2). Actuarial rates of freedom from any operation on the aorta or the aortic graft at 5 and 10 years were $87 \%$ and $37 \%$, respectively (Figure 3 ). The reoperative procedures performed are shown in Table 4 . Fifteen of the 18 patients in whom 20 reoperations were required had Marfan or Loeys-Dietz syndrome. The most common procedure was open or endovascular repair for 
TABLE 1. Preoperative patient characteristics

\begin{tabular}{lc}
\multicolumn{1}{c}{ Characteristics } & $\begin{array}{r}\text { No. }(\%) \text { of patients } \\
(\mathbf{N}=\mathbf{6 9})\end{array}$ \\
\hline Comorbidities & $30(43)$ \\
Marfan, Loeys-Dietz syndrome & $49(71)$ \\
Hypertension & $22(32)$ \\
Hyperlipidemia & $17(25)$ \\
Chronic obstructive pulmonary disease & $10(15)$ \\
Coronary artery disease & $2(3)$ \\
Diabetes & $22(32)$ \\
Peripheral vascular disease & $13(19)$ \\
Previous transient ischemic attach or stroke & $29(42)$ \\
History of smoking & $9(13)$ \\
Creatinine >1.5 mg/dL & \\
Previous operations & 53 \\
Ascending aortic repair & 21 \\
Aortic arch repair & 14 \\
Descending thoracic aortic repair & 8 \\
Abdominal aortic repair & 8 \\
Coronary artery bypass graft or percutaneous & \\
$\quad$ coronary intervention & \\
Aortic valve procedure & 44 \\
Mitral valve replacement & 3 \\
Atrial septal defect & $13(22)$ \\
Saphenous vein graft to renal artery & 1 \\
Extent of thoracoabdominal aortic dissection & \\
Crawford extent I & \\
Crawford extent II & \\
Crawford extent III & $19)$ \\
\hline & \\
&
\end{tabular}

patch aneurysms of the intercostal or visceral/renal arteries. ${ }^{4}$ Nine of the 10 patients who required these procedures had Marfan or Loeys-Dietz syndrome. No reoperations on the aortic graft or the renal or visceral arteries have been required among the patients who had implantation of a branched graft.

TABLE 2. Cardiopulmonary perfusion data

\begin{tabular}{lcl}
\hline & $\begin{array}{c}\text { Mean } \pm \text { standard } \\
\text { deviation }\end{array}$ & Range \\
\hline Time (min) & & \\
$\quad$ Cardiopulmonary bypass & $197.1 \pm 43.4$ & $90-307$ \\
Cooling & $40.6 \pm 12.4$ & $22-75$ \\
Circulatory arrest & $30.8 \pm 11.0$ & $8-61$ \\
Low-flow hypothermic bypass & $62.7 \pm 28.9$ & $16-160$ \\
Hypothermic ventricular fibrillation & $128.6 \pm 34.6$ & $41-230$ \\
Spinal cord ischemia & $78 \pm 26.2$ & $30-172$ \\
Left renal ischemia & $111.1 \pm 38.8$ & $16-202$ \\
Right renal ischemia & $108.3 \pm 37.5$ & $16-200$ \\
Celiac ischemia & $119 \pm 39.4$ & $16-215$ \\
Superior mesenteric artery ischemia & $111.7 \pm 38.7$ & $16-230$ \\
Rewarming & $81.2 \pm 22.7$ & $49-154$ \\
Temperature $\left({ }^{\circ} \mathrm{C}\right)$ & & \\
Lowest nasopharyngeal & $15 \pm 2.4$ & $11.8-24$ \\
Lowest bladder/rectal & $18.9 \pm 2.6$ & $12.3-26.5$ \\
\hline
\end{tabular}

TABLE 3. Extent of repair and associated mortality and morbidity

\section{0-d Spinal cord}

Crawford extent mortality, Stroke, ischemic injury, New dialysis,

\begin{tabular}{lcccc}
\multicolumn{1}{c}{ of repair } & $\mathbf{n}(\%)$ & $\mathbf{n}(\%)$ & $\mathbf{n}(\%)^{*}$ & $\mathbf{n}(\%)^{*}$ \\
\hline Type I $(\mathrm{n}=13)$ & 0 & 0 & 0 & 0 \\
Type II $(\mathrm{n}=41)$ & 3 & 1 & 1 & 2 \\
Type III $(\mathrm{n}=15)$ & 1 & 1 & 3 & 1 \\
Total $(\mathrm{n}=69)$ & $4(5.8)$ & $2(3.0)$ & $4(6.1)$ & $3(4.5)$ \\
\hline
\end{tabular}

*Based on 66 operative survivors.

\section{DISCUSSION}

Our cumulative experience with CPB and HCA for the management of thoracic and thoracoabdominal aortic disease has confirmed the safety and efficacy of the technique. $^{1,2}$ The present study demonstrates its effectiveness for the subset of patients with chronic thoracoabdominal aortic dissection. Corvera and Fehrenbacher ${ }^{5}$ have also reported satisfactory outcomes using this technique for patients with chronic thoracic and thoracoabdominal aortic dissection. Other groups, using differing techniques, have reported similar or higher early mortality rates and comparable rates of major morbidity for open repair of chronic descending aortic dissections. ${ }^{6-8}$ However, these series included patients with both thoracic and thoracoabdominal procedures, and the subgroups were not analyzed separately. Several of these reports have also noted the need for subsequent open and endovascular vascular procedures. Pujara and colleagues ${ }^{7}$ reported a $79 \%$ rate of freedom from reoperation at 5 years among patients treated with open repair. They observed, as we did, that these procedures involved the aorta both proximal and distal to the operated segment, as well as the operated segment.

Early in our experience with Crawford extent II and III disease, the renal and visceral arteries were attached to the aortic graft with a full-thickness cuff of aortic tissue. Patch aneurysms and false aneurysms requiring reoperation

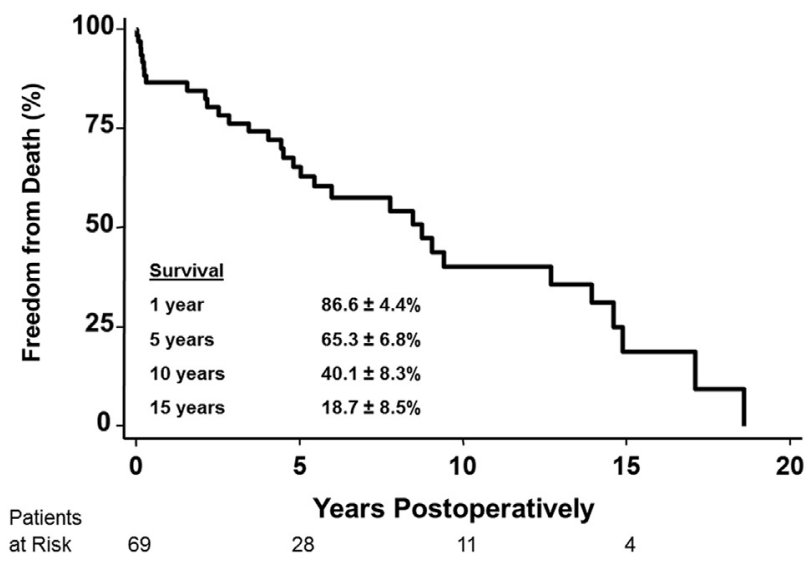

FIGURE 1. Long-term survival after repair of chronic thoracoabdominal aortic dissection. 


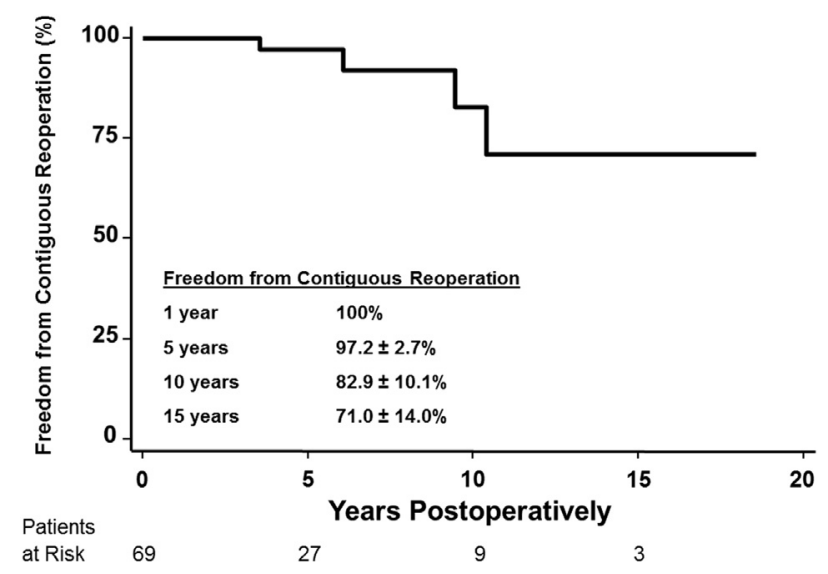

FIGURE 2. Freedom from reoperation on the contiguous proximal or distal aorta after repair of chronic of chronic thoracoabdominal aortic dissection.

developed in some of these patients. Since adopting the use of branched grafts to restore flow to the visceral and renal arteries, these complications have been eliminated, and patency of the branch grafts has been excellent. ${ }^{4}$ Intercostal and lumbar arteries that were preserved were also attached to the aortic graft with a cuff of aortic tissue. A substantial percentage of these patients, particularly those with Marfan or Loeys-Dietz syndrome, developed patch aneurysms that required open or endovascular repair from 7 months to 14.4 years after the initial procedure (Table 4). All patients survived the reoperative procedure. We continue to attach patent lumbar and lower intercostal arteries with a cuff of aortic tissue, when technically feasible, to maintain flow to the spinal cord, particularly in younger patients who may not have developed adequate collateral pathways to supply the spinal cord. However, because of the propensity for development of patch aneurysms, continued surveillance with imaging is essential. Although interposition grafts have been used as an alternative method to preserve patent intercostal arteries, Omura and colleagues ${ }^{9}$ reported

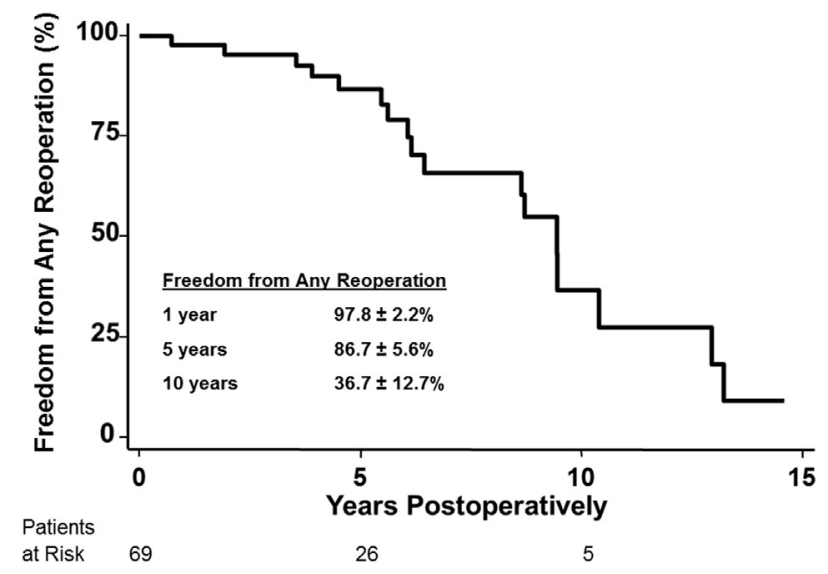

FIGURE 3. Freedom from any operation on the aorta or the aortic graft after repair of chronic thoracoabdominal aortic dissection.
TABLE 4. Subsequent aortic procedures in 18 patients*

\begin{tabular}{lc}
\hline \multicolumn{1}{c}{ Aortic segment } & $\begin{array}{c}\text { No. }(\%) \text { of reoperations } \\
(\mathbf{N}=\mathbf{2 0})\end{array}$ \\
\hline Aortic root & $3(4.3)$ \\
Proximal contiguous aorta & $3(4.3)$ \\
Aortic graft patch or other false aneurysm $\dagger$ & $10(14.5)$ \\
Distal contiguous aorta & $3(4.3)$ \\
Abdominal aorta & $1(1.5)$ \\
Total & $20(29)$ \\
\hline 15 of the18 patients had Marfan or Loeys-Dietz syndrome. $\dagger 9$ of the 10 patients had \\
Marfan or Loeys-Dietz syndrome.
\end{tabular}

a significantly higher patency rate with the intercostal patch technique, $90.8 \%$ (109 of 120 arteries) compared with $74.2 \%$ (256 of 345 arteries) with the graft interposition technique $(P<.01)$.

Uncertainty remains regarding the role of endovascular stent grafting and hybrid techniques for the management of chronic thoracoabdominal aortic dissection, and the results to date have been variable. A meta-analysis of outcomes from 39 studies of distal aortic dissection involving 197 patients treated with endovascular grafts reported a 30-day mortality of 3.2\% and 2-year survival of $91 \% .^{10}$ The rates of overall, major, and procedure-related complications (including retrograde aortic dissection) were $9.1 \%$, $7.9 \%$ and $8.3 \%$, respectively. In a subsequent systematic review of midterm outcomes after endovascular repair that involved 17 studies of 567 patients, the midterm mortality was $9.2 \%$. ${ }^{11}$ Reintervention rates ranged from $0 \%$ to $60 \%$ in those studies with a median follow-up of 31 months. Distal aneurysm formation or continued false aneurysm perfusion with aneurysmal dilatation developed in $7.8 \%$ of patients. In a series of 76 patients reported from the Cleveland Clinic, the 30-day mortality was 5\% and the 3 -year survival was $80 \%{ }^{12}$ During a mean follow-up interval of 24 months, 19 reinterventions were required in 17 patients, more than half of which entailed aortic repairs distal to the endograft. Three patients sustained a retrograde aortic dissection, prosthetic collapse occurred in 2 patients, and most importantly, enlargement of the aorta distal to the stent occurred in 9 patients. The intervention-free rate was $73 \%$ at 3 years. Freedom from death or intervention at 3 years was $59 \%$. These results suggest that endovascular repair may not be the optimal method of management for patients with extensive thoracoabdominal aortic dissection, particularly when the aneurysmal enlargement extends into the infradiaphragmatic segment of the aorta.

Hybrid procedures represent another alternative for repair of chronic thoracoabdominal aortic dissection. However, in a recently published meta-analysis of 19 studies of 660 patients who had hybrid repair of thoracoabdominal aortic aneurysms, only $7.8 \%$ of the procedures involved patients with aortic dissection, and the outcomes of this subset were not analyzed separately. ${ }^{13}$ Early mortality for the 
entire cohort was $12.6 \%$, and the overall rate of spinal cord ischemia was $3.4 \%$. The overall rates of permanent renal failure, mesenteric ischemia, and severe cardiopulmonary complications were $10.4 \%, 4.6 \%$, and $7.8 \%$, respectively. The overall rate of renal and visceral artery graft patency during a mean follow-up of 26.2 months was $94.7 \%$. Experience with fenestrated and branched endovascular aortic repair for chronic thoracoabdominal aortic dissections remains limited. ${ }^{14}$

Because of selection bias, differing operative techniques, and lack of long-term follow-up, particularly for the more recently implemented endovascular and hybrid techniques, uncertainty regarding the optimal method of management will persist until the results of randomized trials comparing well-matched patients in whom standardized open and endovascular techniques are used become available.

\section{Limitations}

The present study was observational in nature and involved retrospective review of prospectively collected data in a referral-based tertiary care center. Surgical techniques evolved during the 18 year time period of the study. However, long-term follow-up, including periodic imaging studies, was available for most patients.

\section{CONCLUSIONS}

Open repair of chronic thoroacoabdominal aortic dissection using total hypothermic CPB and HCA can be accomplished with acceptable mortality and morbidity rates that are comparable with those reported for endovascular or hybrid techniques. Open repair should remain a viable and primary option for repair of this condition until the long-term effectiveness of the alternative methods of treatment is clearly established.

\section{References}

1. Kulik A, Castner CF, Kouchoukos NT. Replacement of the descending thoracic aorta: contemporary outcomes using hypothermic circulatory arrest. Cardiovasc Surg. 2010;139:249-55.

2. Kulik A, Castner CF, Kouchoukos NT. Outcomes after thoracoabdominal aortic aneurysm repair with hypothermic circulatory arrest. J Thorac Cardiovasc Surg. 2011:141:953-60.

3. Kouchoukos NT, Masetti P, Castner CF. Use of presewn multiple branched graft in thoracoabdominal aortic aneurysm repair. J Am Coll Surg. 2005;201:646-9.

4. Kulik A, Allen BT, Kouchoukos NT. Incidence and management of intercostal patch aneurysms after repair of thoracoabdominal aortic aneurysms. $J$ Thorac Cardiovasc Surg. 2009;138:352-8.

5. Corvera JS, Fehrenbacher JW. Open repair of chronic aortic dissections using deep hypothermia and circulatory arrest. Ann Thorac Surg. 2012;94:78-83.

6. Zoli S, Etz CD, Roder F, Mueller CS, Brenner RM, Bodian CA, et al. Long-term survival after open repair of chronic distal aortic dissection. Ann Thorac Surg. 2010;89:1458-66.

7. Pujara AC, Roselli EE, Hernandez AV, VargasAbello LM, Burke JM, Svensson LG, et al. Open repair of chronic distal aortic dissection in the endovascular era: implications for disease management. J Thorac Cardiovasc Surg. 2012;144:866-73.

8. Conway AM, Sadek M, Lugo J, Pillai JB, Pellet Y, Panagopoulos G, et al. Outcomes of open surgical repair for chronic type B aortic dissections. J Vasc Surg. 2014;59:1217-23.

9. Omura A, Yamanaka K, Miyahara S, Sakamoto T, Inoue T, Okada K, et al. Early patency rate and fate of reattached intercostal arteries after repair of thoracoabdominal aortic aneurysms. J Thorac Cardiovasc Surg. 2014;147:1861-7.

10. Eggebrecht $H$, Nienaber CA, Neuhauser M, Baumgart D, Kische S, Schmermund A, et al. Endovascular stent-graft placement in aortic dissection: a meta-analysis. Eur Heart J. 2006;27:489-98.

11. Thrumurthy SG, Karthikesalingam A, Patterson BO, Holt PJE, Hinchliffe RJ, Loftus IM, et al. A systematic review of mid-term outcomes of thoracic endovascular repair (TEVAR of chronic type B aortic dissection. Eur J Vasc Endovasc Surg. 2011;42:632-47.

12. Kang WC, Greenvert RK, Mastracci TM, Eagleton MJ, Hernandez AV Pujara AC, et al. Endovascular repair of complicated chronic distal aortic dissections: intermediate outcomes and complications. J Thorac Cardiovasc Surg. 2011;142:1074-83.

13. Canaud L, Karthikesalingam A, Jackson D, Cresswell L, Cliff M, Markar SS et al. Clinical outcomes of single versus staged hybrid repair for thoracoabdominal aortic aneurysm. J Vasc Surg. 2013;58:1192-200.

14. Kitagawa A, Greenberg RK, Eagleton MJ, Mastracci TM, Roselli EE Fenestrated and branched endovascular aortic repair for chronic type B aortic dissection with thoracoabdominal aneurysms. J Vasc Surg. 2013;58:625-34. 